\title{
Analgesic Effects of Navigated Repetitive Transcranial Magnetic Stimulation in Patients With Acute Central Poststroke Pain
}

\author{
Chen-Guang Zhao · Wei Sun · Fen Ju · Shan Jiang · Hong Wang • \\ Xiao-Long Sun $\cdot$ Xiang Mou $\cdot$ Hua Yuan (D)
}

Received: February 12, 2021 / Accepted: March 30, 2021 / Published online: April 17, 2021

(C) The Author(s) 2021, corrected publication 2021

\begin{abstract}
Introduction: Central poststroke pain (CPSP) develops commonly after stroke, which impairs the quality of life, mood, and social functioning. Current pharmacological approaches for the treatment of CPSP are not satisfactory. Repetitive transcranial magnetic stimulation (rTMS) is a noninvasive technique which has been recommended for the treatment of chronic CPSP. However, few studies have evaluated the analgesic effects of rTMS in patients with acute neuropathic pain after stroke.

Methods: We evaluated the analgesic effects of rTMS applied over the upper extremity area of the motor cortex (M1) in patients with acute CPSP. Forty patients were randomized to receive either rTMS $(10 \mathrm{~Hz}, 2000$ stimuli) $(n=20)$ or a sham intervention $(n=20)$ for 3 weeks. The Numeric Rating Scale (NRS), Short-form McGill Pain Questionnaire-2 (SF-MPQ-2, Chinese
\end{abstract}

Chen-Guang Zhao and Wei Sun contributed equally to this article as co-first authors.

C.-G. Zhao - W. Sun - F. Ju - H. Wang - X.-L. Sun ·

X. Mou $\cdot$ H. Yuan $(\bowtie)$

Department of Rehabilitation Medicine, Xijing

Hospital, Fourth Military Medical University, Xi'an, China

e-mail: yuanhua_fmmu@hotmail.com

S. Jiang

Department of Rehabilitation Medicine, China-

Japan Friendship Hospital, Beijing, China version), Hamilton Anxiety Scale (HAM-A), Hamilton Depression Scale (HAM-D), brainderived neurotrophic factor (BDNF) levels, and motor-evoked potentials (MEP) were analyzed at baseline, 3 days, 1 week, 2 weeks, and 3 weeks.

Results: Significant treatment-time interactions were found for pain intensity. Compared with the sham group, the NRS and SF-MPQ-2 scores were significantly lower on the seventh day of treatment in the rTMS group $(P<0.001$, Cohen's $d=1.302) \quad(P=0.003, \quad$ Cohen's $d=0.771)$, and this effect lasted until the third week $(P=0.001$, Cohen's $d=0.860)(P=0.027$, Cohen's $d=0.550)$. The HAM-A and HAM-D scores did not change in the rTMS group when compared with the sham group $(P=0.341$, Cohen's $d=0.224) \quad(P=0.356, \quad$ Cohen's $\mathrm{d}=0.217)$. The serum BDNF levels were significantly higher in the treated group $(P=0.048$, Cohen's $d=-0.487)$, and the resting motor threshold (RMT) decreased by $163.65 \%$.

Conclusion: Our findings indicate that rTMS applied over the upper extremity area of the motor cortex can effectively alleviate acute CPSP, possibly by influencing cortical excitability and serum BDNF secretion.

Trial Registration: This trial is registered with Clinical Trial Registry of China: Reg. No. ChiCTR-INR-17012880. 
Keywords: BDNF; Central poststroke pain; Cortical excitability; Motor cortex stimulation; Repetitive transcranial magnetic stimulation

\section{Key Summary Points}

Why carry out this study?

Central poststroke pain (CPSP) typically develops within 1 to 6 months after a stroke, and seriously impairs the patient's quality of life, mood, and social functioning.

Current pharmacological approaches for the treatment of CPSP have failed to provide adequate pain relief.

We aimed to evaluate the analgesic effects of repetitive transcranial magnetic stimulation (rTMS) in patients with acute CPSP.

\section{What was learned from the study?}

High-frequency $(10 \mathrm{~Hz})$ rTMS applied over the upper extremity area of the motor cortex can effectively alleviate acute CPSP.

rTMS may also relieve depression and anxiety after CPSP.

The analgesic effects of rTMS may be associated with enhanced cortical excitability and increased secretion of brain-derived neurotrophic factor.

\section{DIGITAL FEATURES}

This article is published with digital features, including a summary slide, to facilitate understanding of the article. To view digital features for this article go to https://doi.org/10.6084/ m9.figshare.14333807.

\section{INTRODUCTION}

Stroke is one of the most common causes of disability and death among adults [1]. During the rehabilitation phase, some stroke survivors develop central neuropathic pain as a result of the cerebrovascular lesion itself, a syndrome known as central poststroke pain (CPSP). CPSP develops most commonly within 1 to 6 months after a stroke [2-5], which is also the critical period for functional recovery. CPSP impairs the quality of life, mood, and social functioning of the patient, negatively affecting the rehabilitation process. Even worse, motor function can be impaired by pain; in other words, CPSP can interfere with motor learning capacities and/or motor performance [6]. The incidence of CPSP in stroke patients varies significantly, with a prevalence ranging from $1 \%$ to $35.4 \%$, according to published studies $[3,4,6-8]$. The clinical characteristics of CPSP commonly include allodynia and dysesthesia [9], which can be variously described as a burning, aching, pricking, freezing, squeezing, shooting, or prickling sensation.

Current pharmacological approaches for the treatment of CPSP, including antidepressants, anticonvulsants, and antiepileptics, among others [10-12], have failed to provide adequate pain relief [13], prompting clinicians to search for possible alternatives. Repetitive transcranial magnetic stimulation (rTMS) is a noninvasive technique involving the application of rapidly changing magnetic fields over an objective encephalic region to influence neural excitability. In this regard, high-frequency rTMS (usually $5-20 \mathrm{~Hz}$ ) over an objective encephalic region has been recommended for the treatment of CPSP [14, 15], including pain produced by spinal cord injury (SCI), stroke, or multiple sclerosis (MS). The pain reduction ranged between $10 \%$ and $40 \%$.

Levels of brain-derived neurotrophic factor (BDNF), a traditional neuronal modulator, have been reported to show a close relationship with neuropathic pain and neuronal plasticity. In a previous study, we showed that serum BDNF levels increased after 3 weeks of high-frequency rTMS in patients with SCI. These findings were 
in agreement with those of Agnol et al., who reported increased secretion of BDNF after ten sessions of rTMS [16], although others did not find similar changes [17].

Previous studies have focused on the chronic phase of CPSP. However, few studies have evaluated rTMS in patients with acute CPSP. Therefore, we used several questionnaires to assess pain and mood before and after treatment with rTMS. Furthermore, we evaluated whether neurotrophic factor levels and neurophysiological parameters, which have a close relationship with CPSP, were also influenced by rTMS.

\section{METHODS}

\section{Study Design and Ethical Considerations}

This was a prospective, randomized, doubleblind, two-group parallel clinical study. It was approved by the Institutional Ethics Committee of Xijing Hospital, Fourth Military Medical University (KY20162079), which adhere to the tenets of the Declaration of Helsinki, and was registered in the Chinese Clinical Trial Registry (Reg. No. ChiCTR-INR-17012880). Patients provided informed consent to participate in the trial. Written informed consent was received from the patients for publication of the data and any accompanying images.

Using the $G^{*}$ Power v.3.1.9.2 statistical tool to achieve a statistical power of $85 \%$ with statistical significance at $P<0.05$ (two-tailed test) and an effect size of $r=0.4$, a minimum sample size of 36 patients was required for the present trial.

A computer random number generator assigned patients to the rTMS or sham group using a block randomization strategy (Fig. 1). Before the recruitment phase, opaque envelopes containing the protocol materials and group allocation were prepared, sealed, and numbered sequentially. After the patient agreed to participate in the trial, the envelope in the sequence was opened by the coordinator, who was not involved with the patient's intervention.

All investigators were rehabilitation science majors with more than 3 years of professional experience. To guarantee that the study was

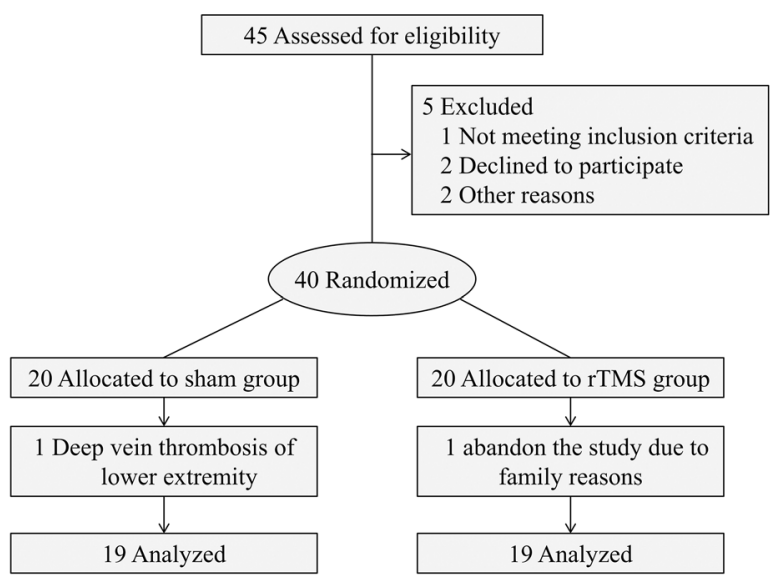

Fig. 1 CONSORT diagram showing study enrollment

performed in a double-blind manner, two independents, specially trained physicians, evaluated the outcome. Participants, as well as those responsible for performing the procedure, were unaware of the group to which the patient had been allocated. Besides rTMS, conventional physical therapy (PT), occupational therapy (OT), and medical treatments (e.g., management of high blood pressure) were also provided to the participants.

\section{Participants}

Participants were enrolled consecutively between October 1, 2016, and December 31, 2018 , from the inpatient population of the department of rehabilitation of Xijing Hospital.

The inclusion criteria were as follows: (1) first stroke episode, (2) confirmed CPSP diagnosis, sensory disturbance corresponding to the cerebral lesion, (3) admitted $\leq 6$ months after stroke onset, $(4) \leq 2$ weeks after pain onset [11], (5) age between 30 and 70 years, (6) ability to comprehend instructions (Mini-Mental State Examination score $\geq 22$ ), (7) absence of aphasia, and (8) completion of signed informed consent by the patient or his/her proxy.

The exclusion criteria were as follows: (1) history of seizure, (2) history of craniotomy, (3) implanted stimulator, including pacemakers, or other metallic objects in any part of the body, (4) history of depression or anxiety before stroke, (5) current use of any medications that 
interfere with rTMS, (5) pain anywhere in the body due to other diseases, or (6) active malignancy or pregnancy.

\section{rTMS Procedure}

rTMS was applied with a CCY-1 stimulator (Yiruide Medical Equipment Company, China) using a figure-of-eight coil, following the safety guidelines and protocols for rTMS. Participants were asked to lie comfortably on the examination couch in a quiet room. We determined the resting motor threshold (RMT) as follows: The target muscle was the first dorsal interosseous (FDI) of the hand, contralateral to the infarct. After the skin was prepared, two $\mathrm{Ag}-\mathrm{AgCl}$ recording surface electromyography electrodes (approximately $1 \mathrm{~cm}^{2}$ ) were placed on the venter musculi. Electromyography (EMG) signals were filtered, amplified, displayed, and stored in a computer. The RMT was defined as the lowest stimulation intensity capable of eliciting motorevoked potentials (MEPs) $\geq 50 \mu \mathrm{V}$ peak-to-peak amplitude in at least five of ten consecutive stimulations (50\%). If the RMT in the affected hemisphere $(\mathrm{AH})$ could not be detected, we measured the RMT in the unaffected hemisphere (UH). In the active rTMS stimulation group, rTMS was applied over the motor cortical area (M1) corresponding to the painful zone at a frequency of $10 \mathrm{~Hz}$, as 15 pulse trains $(1.5 \mathrm{~s})$, with intertrain intervals of $3 \mathrm{~s}$ (total of 1500 pulses) and at an intensity of $80 \%$ of the RMT $(\mathrm{AH})$, or $100 \%(\mathrm{UH})$ when the RMT could not be detected in the AH. The sham stimulation was delivered using a coil identical to the one in the active group (same shape and color), but with no magnetic stimulation output (only emitting the same sound). Patients in the active rTMS and sham groups received stimulation once a day, 6 days per week, for a total of 3 weeks.

\section{Neuronavigation}

We used a neuronavigation system (visor2, ANT Neuro b.v., Enschede, Netherlands) to guide the magnetic stimulation, as described previously [18]. The system generated a three-dimensional reconstruction of the patient's brain based on magnetic resonance imaging (MRI) data obtained before the first session. An infrared camera allowed the operator to precisely position the coil over the target area under real-time visualization (Fig. 2).

\section{Blood Collection and Measurement of Serum BDNF Levels}

Blood samples were collected in EDTA-treated tubes (BD, Franklin Lakes, NJ, USA), anonymized, immediately frozen at $-20^{\circ} \mathrm{C}$, transported to the key laboratory, and stored at $-80^{\circ} \mathrm{C}$ until they were subjected to analysis. Serum BDNF concentrations were measured using enzyme-linked immunosorbent assay (ELISA) kits (Multi Sciences, Catalog No. EK11272, lower detection limit $=1.41 \mathrm{pg} / \mathrm{ml}$ ), according to the manufacturer's instructions.

\section{Outcome Measurements}

Pain intensity was evaluated at different time points: before intervention (T0), and 3 days (T1), 1 week (T2), 2 weeks (T3), and 3 weeks (T4) afterwards. Mood assessments and biochemical and neurophysiological measurements were performed only at T0 and T4. Figure 3 shows the study timetable.

The primary outcomes were as follows:

1. Numeric rating scale (NRS). The intensity of pain was measured by the NRS, which consists of an 11-point scale, where 0 indicates no pain and 10 is the worst pain imaginable. The NRS has been recommended by the Initiative on Methods, Measurement, and Pain Assessment in Clinical Trials (IMMPACT) consensus group to measure pain in clinical trials [19].

2. Short-form McGill Pain Questionnaire-2 (Chinese Edition) (SF-MPQ-2). It includes 20 items that assess four aspects of pain, each item rated on a 0-10 NRS scale [20]. The four subscales have been described before: six items assess continuous pain, six items assess intermittent soreness, six items assess neuropathic pain, and four items assess the affective sphere. Scores on 

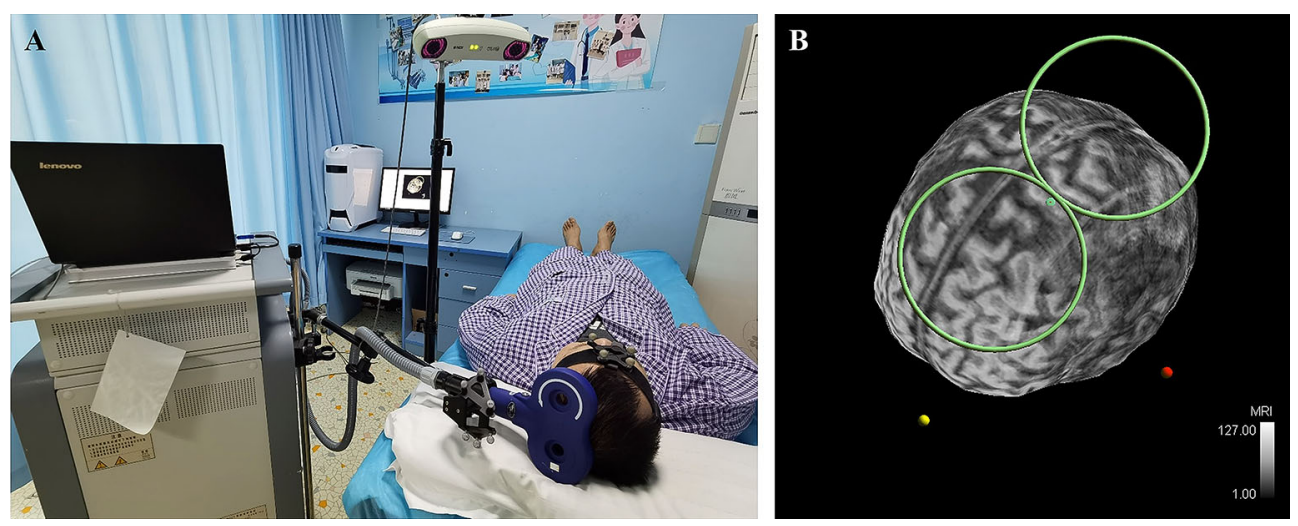

Fig. 2 Neuronavigation-guided rTMS. a Image of rTMS neuronavigation system used on patients with stroke. b Real-time visualization of magnetic coil stimulating the upper extremity area of the M1 cortex

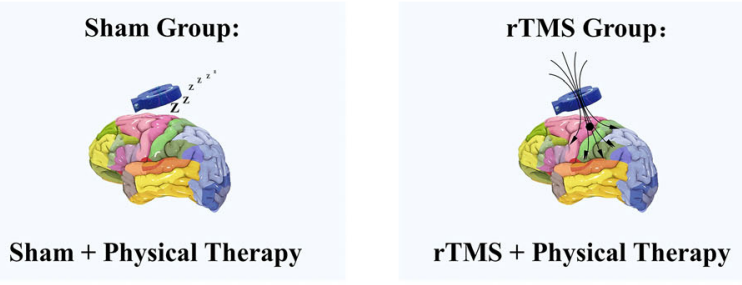

\section{Treatments}

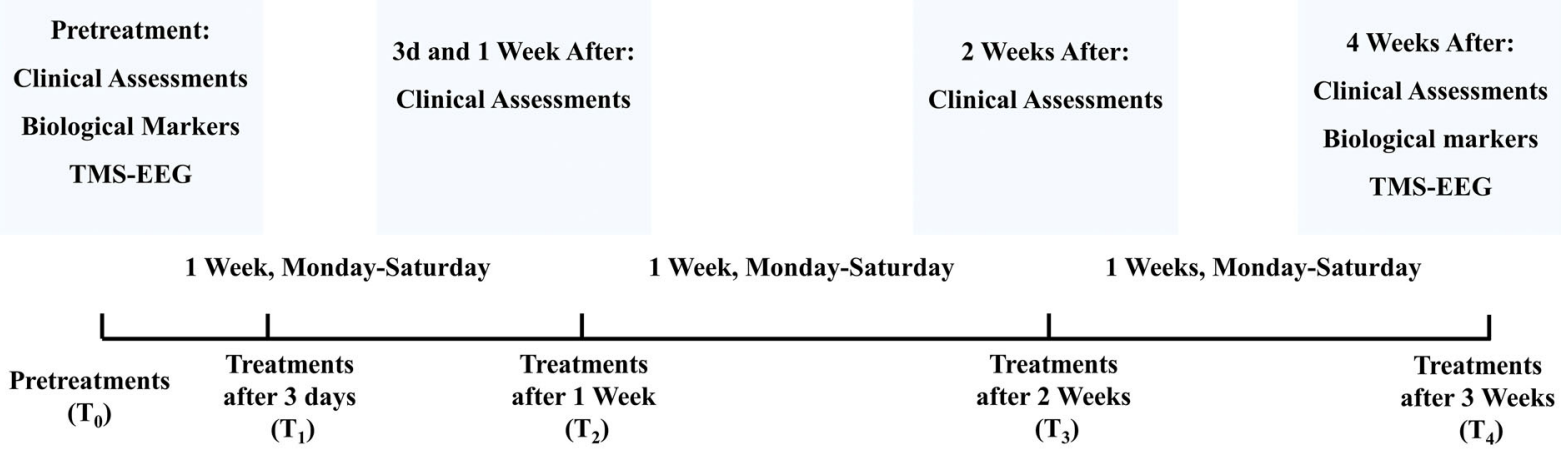

Fig. 3 Schematic diagram of the experimental design. Pain intensity was evaluated by means of NRS and SF-MPQ-2 questionnaires at five different time points: T0 (before treatment), T1 (on the third day), T2 (after 1 week), T3 (after 2 weeks), and T4 (after 3 weeks). Mood changes (HAM-A, HAM-D), neurotrophic factor levels (BDNF), and neurophysiological parameters (RMT, MEP latency, MEP maximal amplitudes) were evaluated only at T0 and T4

these four aspects were added to obtain the final score. A higher score represents more severe pain.

The secondary outcomes were as follows:

1. Anxiety was evaluated by means of the Hamilton Anxiety Scale (HAM-A). HAM-A includes 14 items; each item is scored from 0 to 4 , with a higher score reflecting more severe anxiety [21].

2. Depression was assessed by means of the Hamilton Depression Scale (HAM-D) $[22,23]$. It consists of 17 items; each item is scored from 0 (not present) to 7 (severe), 
with a higher total score indicating more severe depression.

3. Serum BDNF levels.

4. Neurophysiological parameters, including RMT, MEP amplitude, and MEP latency. We applied single-pulse TMS at 70-85\% intensity of the machine export threshold over the affected hemisphere to measure motor cortex excitability. MEP latency and amplitude were calculated based on five waves showing large amplitude and good repeatability. The MEP amplitude is defined as the peak-to-peak amplitude $(\mathrm{mV})$ in the EMG response of the contralateral FDI. The MEP latency is defined as the interval between the start of the single-pulse TMS on M1 and the initiation of the EMG response (ms) in the contralateral target muscle. A TMS-elicited MEP was categorized as present or absent [24-26].

\section{Statistical Analysis}

SPSS software (version 20.0; IBM Corp., Armonk, NY, USA) was used for statistical analysis. The effects of rTMS on pain and mood assessments, as well as on biochemical and neurophysiological parameters, were analyzed by means of repeated-measures analysis of variance (rmANOVA), with time as the withinsubjects factor and treatment as the betweensubjects factor. Bonferroni adjustment was performed as post hoc analysis for multiple comparisons. A $P$ value less than 0.05 was considered statistically significant. We calculated Cohen's $d$ and $\eta^{2}$ as measures of effect sizes in this study. The interpretation of Cohen's $d$ and $\eta^{2}$ was described in our previous paper [18]. The percentage of change within each individual was calculated as follows: [(Post-rTMS - Pre-rTMS Score)/(Pre-rTMS Score) $] \times 100$.

\section{RESULTS}

A total of 40 patients with CPSP were finally enrolled in the study. They were randomly assigned to the rTMS group $(n=20)$ or the sham group $(n=20)$. In the sham group, one patient had to abandon the study for family reasons, while another patient with deep vein thrombosis of the lower extremity in the rTMS group dropped out of the trial. The remaining 38 patients completed the 3 -week trial. There were no significant differences between the two groups in the mean (SD) age (sham: 48.95 [11.51] VS rTMS 50.16 [11.34], $P=0.746$ ), time since injury (sham: 12.21 [5.01] VS rTMS 10.63 [5.77], $P=0.374$ ), or pain duration (sham: 6.47 [12.57] VS rTMS 6.00 [3.07], $P=0.609$ ). No significant differences were found between the two groups in terms of demographic variables or clinical characteristics (Table 1). Of the 38 participants, three reported short periods of numbness in the scalp or twitching of facial muscles during the rTMS procedure, but no serious adverse effects were observed.

\section{Primary Outcome}

\section{Pain Scores}

NRS The within-subject effects in the rTMS group were significant $\left(P<0.001 \eta^{2}=0.664\right)$. The pain intensity started to decrease on the third day after the intervention $(P=0.073$, Cohen's $d=0.694$ ) and was lowest on the seventh day $(P<0.001$, Cohen's $d=1.906)$ when compared with baseline. Although the NRS score increased slightly afterwards, the pre-post effects remained significant 3 weeks after the intervention $(P<0.001$, Cohen's $d=1.289)$. In contrast, there were no significant within-subject effects in the sham group $(P=0.301$, $\left.\eta^{2}=0.065\right)$. The interaction between time and intervention was significant $(P<0.001$, $\left.\eta^{2}=0.551\right)$. The NRS score in the rTMS group was significantly lower than in the sham group on the seventh day $(P<0.001$, Cohen's $d=1.302)$, and this effect lasted until the third week $(P=0.001$, Cohen's $d=0.860)$. Pain intensity decreased by $20.29 \%$ in the rTMS group and by $0.49 \%$ in the sham group (Table 2 , Fig. 4a).

SF-MPQ-2 Compared with baseline SF-MPQ-2 scores (before intervention), the lowest scores in the rTMS group were observed on the seventh 
Table 1 Demographic and clinical information of patients

\begin{tabular}{|c|c|c|c|c|c|c|c|c|}
\hline No. & Gender & $\begin{array}{l}\text { Age } \\
\text { (years) }\end{array}$ & $\begin{array}{l}\text { Time since } \\
\text { injury }\end{array}$ & $\begin{array}{l}\text { Pain } \\
\text { duration } \\
\text { (days) }\end{array}$ & $\begin{array}{l}\text { Type of } \\
\text { stroke }\end{array}$ & Lesion & Pain location & $\begin{array}{l}\text { Quality of } \\
\text { pain }\end{array}$ \\
\hline 1 & $\mathrm{M}$ & 30 & $\begin{array}{l}2 \text { months and } \\
2 \text { weeks }\end{array}$ & 2 & I & Right cerebellum & $\begin{array}{l}\text { Lower } \\
\text { extremity }\end{array}$ & $\begin{array}{l}\text { Freezing, } \\
\text { squeezing }\end{array}$ \\
\hline 2 & $\mathrm{M}$ & 41 & 2 months & 6 & $\mathrm{H}$ & Left thalamus & $\begin{array}{l}\text { Hemibody } \\
\text { with face }\end{array}$ & $\begin{array}{l}\text { Aching, } \\
\text { pricking }\end{array}$ \\
\hline 3 & $\mathrm{M}$ & 63 & 5 months & 8 & $\mathrm{H}$ & Right thalamus & $\begin{array}{l}\text { Lower } \\
\text { extremity }\end{array}$ & $\begin{array}{l}\text { Numbness, } \\
\text { freezing }\end{array}$ \\
\hline 4 & $\mathrm{~F}$ & 61 & 2 weeks & 3 & I & $\begin{array}{l}\text { Right basal } \\
\text { ganglia }\end{array}$ & $\begin{array}{l}\text { Upper } \\
\text { extremity }\end{array}$ & $\begin{array}{l}\text { Freezing, } \\
\text { pressing }\end{array}$ \\
\hline 5 & $\mathrm{M}$ & 52 & $\begin{array}{l}1 \text { month and } \\
2 \text { weeks }\end{array}$ & 5 & I & Left thalamus & $\begin{array}{l}\text { Hemibody } \\
\text { without face }\end{array}$ & $\begin{array}{l}\text { Freezing, } \\
\text { squeezing }\end{array}$ \\
\hline 6 & M & 68 & 3 months & 6 & $\mathrm{H}$ & Right thalamus & $\begin{array}{l}\text { Lower } \\
\text { extremity }\end{array}$ & $\begin{array}{l}\text { Pricking, } \\
\text { burning }\end{array}$ \\
\hline 7 & M & 56 & $\begin{array}{l}3 \text { months and } \\
2 \text { weeks }\end{array}$ & 8 & $\mathrm{H}$ & Left frontal lobe & $\begin{array}{l}\text { Upper } \\
\text { extremity }\end{array}$ & Numbness \\
\hline 8 & $\mathrm{M}$ & 59 & 2 months & 10 & I & Left thalamus & $\begin{array}{l}\text { Upper } \\
\text { extremity }\end{array}$ & $\begin{array}{c}\text { Numbness, } \\
\text { freezing }\end{array}$ \\
\hline 9 & $\mathrm{~F}$ & 61 & 2 months & 4 & $\mathrm{H}$ & Left basal ganglia & $\begin{array}{l}\text { Hemibody } \\
\text { with face }\end{array}$ & $\begin{array}{l}\text { Burning, } \\
\text { squeezing }\end{array}$ \\
\hline 10 & M & 44 & $\begin{array}{l}4 \text { months and } \\
2 \text { weeks }\end{array}$ & 5 & $\mathrm{H}$ & $\begin{array}{l}\text { Left external } \\
\text { capsule }\end{array}$ & $\begin{array}{l}\text { Upper } \\
\text { extremity }\end{array}$ & $\begin{array}{l}\text { Throbbing, } \\
\text { tingling }\end{array}$ \\
\hline 11 & $\mathrm{~F}$ & 52 & $\begin{array}{l}2 \text { months and } \\
2 \text { weeks }\end{array}$ & 7 & $\mathrm{H}$ & $\begin{array}{l}\text { Right external } \\
\text { capsule }\end{array}$ & $\begin{array}{l}\text { Hemibody } \\
\text { without face }\end{array}$ & $\begin{array}{c}\text { Numbness, } \\
\text { tingling }\end{array}$ \\
\hline 12 & $\mathrm{M}$ & 64 & $\begin{array}{l}2 \text { months and } \\
3 \text { weeks }\end{array}$ & 2 & $\mathrm{H}$ & Left cerebellum & $\begin{array}{l}\text { Upper } \\
\text { extremity }\end{array}$ & $\begin{array}{l}\text { Prickling, } \\
\text { numbness }\end{array}$ \\
\hline 13 & M & 39 & 3 months & 7 & $\mathrm{H}$ & $\begin{array}{l}\text { Right basal } \\
\text { ganglia }\end{array}$ & $\begin{array}{l}\text { Hemibody } \\
\text { without face }\end{array}$ & $\begin{array}{l}\text { Shooting, } \\
\text { squeezing }\end{array}$ \\
\hline 14 & $\mathrm{~F}$ & 62 & 4 months & 5 & $\mathrm{H}$ & Left basal ganglia & $\begin{array}{l}\text { Upper } \\
\text { extremity }\end{array}$ & Squeezing \\
\hline 15 & M & 57 & 3 months & 9 & $\mathrm{I}$ & $\begin{array}{l}\text { Right lateral } \\
\text { periventricular }\end{array}$ & $\begin{array}{l}\text { Upper } \\
\text { extremity }\end{array}$ & $\begin{array}{l}\text { Tingling, } \\
\text { prickling }\end{array}$ \\
\hline 16 & $\mathrm{~F}$ & 63 & $\begin{array}{l}4 \text { months and } \\
2 \text { weeks }\end{array}$ & 4 & I & $\begin{array}{l}\text { Left lateral } \\
\text { periventricular }\end{array}$ & $\begin{array}{l}\text { Hemibody } \\
\text { with face }\end{array}$ & $\begin{array}{l}\text { Pricking, } \\
\text { squeezing }\end{array}$ \\
\hline 17 & $\mathrm{M}$ & 48 & 3 months & 6 & $\mathrm{I}$ & Left basal ganglia & $\begin{array}{l}\text { Upper } \\
\text { extremity }\end{array}$ & $\begin{array}{l}\text { Tingling, } \\
\text { numbness }\end{array}$ \\
\hline 18 & $\mathrm{~F}$ & 44 & 1 month & 2 & $\mathrm{H}$ & Left basal ganglia & Hemibody & Prickling \\
\hline
\end{tabular}


Table 1 continued

\begin{tabular}{|c|c|c|c|c|c|c|c|c|}
\hline No. & Gender & $\begin{array}{l}\text { Age } \\
\text { (years) }\end{array}$ & $\begin{array}{l}\text { Time since } \\
\text { injury }\end{array}$ & $\begin{array}{l}\text { Pain } \\
\text { duration } \\
\text { (days) }\end{array}$ & $\begin{array}{l}\text { Type of } \\
\text { stroke }\end{array}$ & Lesion & Pain location & $\begin{array}{l}\text { Quality of } \\
\text { pain }\end{array}$ \\
\hline 19 & M & 36 & $\begin{array}{l}2 \text { months and } \\
1 \text { week }\end{array}$ & 7 & $\mathrm{H}$ & $\begin{array}{l}\text { Right basal } \\
\text { ganglia }\end{array}$ & $\begin{array}{l}\text { Hemibody } \\
\text { with face }\end{array}$ & $\begin{array}{r}\text { Numbness, } \\
\text { prickling }\end{array}$ \\
\hline 20 & M & 55 & $\begin{array}{l}1 \text { month and } \\
1 \text { week }\end{array}$ & 11 & $\mathrm{H}$ & $\begin{array}{l}\text { Right external } \\
\text { capsule }\end{array}$ & $\begin{array}{l}\text { Upper } \\
\text { extremity }\end{array}$ & Squeezing \\
\hline 21 & $\mathrm{~F}$ & 35 & 2 weeks & 12 & I & $\begin{array}{l}\text { Left lateral } \\
\text { periventricular }\end{array}$ & $\begin{array}{l}\text { Hemibody } \\
\text { without face }\end{array}$ & $\begin{array}{c}\text { Throbbing, } \\
\text { pricking }\end{array}$ \\
\hline 22 & M & 34 & $\begin{array}{l}4 \text { months and } \\
1 \text { week }\end{array}$ & 5 & I & $\begin{array}{l}\text { Left lateral } \\
\text { periventricular }\end{array}$ & $\begin{array}{l}\text { Hemibody } \\
\text { without face }\end{array}$ & $\begin{array}{l}\text { Pricking, } \\
\text { burning }\end{array}$ \\
\hline 23 & M & 47 & 4 months & 9 & I & Right thalamus & $\begin{array}{l}\text { Upper } \\
\text { extremity }\end{array}$ & Tingling \\
\hline 24 & M & 35 & 4 months & 6 & $\mathrm{H}$ & Right frontal lobe & Hemibody & $\begin{array}{l}\text { Aching, } \\
\text { burning }\end{array}$ \\
\hline 25 & $\mathrm{~F}$ & 53 & 1 month & 2 & $\mathrm{H}$ & Right thalamus & $\begin{array}{l}\text { Hemibody } \\
\text { with face }\end{array}$ & $\begin{array}{l}\text { Prickling, } \\
\text { burning }\end{array}$ \\
\hline 26 & $\mathrm{~F}$ & 54 & 2 months & 4 & $\mathrm{H}$ & $\begin{array}{l}\text { Right basal } \\
\text { ganglia }\end{array}$ & $\begin{array}{l}\text { Lower } \\
\text { extremity }\end{array}$ & $\begin{array}{l}\text { Squeezing, } \\
\text { burning }\end{array}$ \\
\hline 27 & $\mathrm{~F}$ & 54 & 2 weeks & 5 & $\mathrm{H}$ & Left frontal lobe & $\begin{array}{l}\text { Hemibody } \\
\text { without face }\end{array}$ & $\begin{array}{l}\text { Prickling, } \\
\text { burning }\end{array}$ \\
\hline 28 & $\mathrm{~F}$ & 70 & 5 months & 7 & I & $\begin{array}{l}\text { Right basal } \\
\text { ganglia }\end{array}$ & $\begin{array}{l}\text { Hemibody } \\
\text { without face }\end{array}$ & $\begin{array}{l}\text { Pricking, } \\
\text { squeezing }\end{array}$ \\
\hline 29 & $\mathrm{~F}$ & 55 & $\begin{array}{l}3 \text { months and } \\
2 \text { weeks }\end{array}$ & 9 & $\mathrm{H}$ & Left basal ganglia & $\begin{array}{l}\text { Upper } \\
\text { extremity }\end{array}$ & $\begin{array}{l}\text { Pricking, } \\
\text { burning }\end{array}$ \\
\hline 30 & $\mathrm{~F}$ & 50 & $\begin{array}{l}5 \text { months and } \\
1 \text { week }\end{array}$ & 4 & I & Left basal ganglia & $\begin{array}{l}\text { Upper } \\
\text { extremity }\end{array}$ & Burning \\
\hline 31 & $\mathrm{~F}$ & 59 & 5 months & 6 & I & Left basal ganglia & $\begin{array}{l}\text { Hemibody } \\
\text { without face }\end{array}$ & $\begin{array}{l}\text { Freezing, } \\
\text { throbbing }\end{array}$ \\
\hline 32 & $\mathrm{~F}$ & 42 & 10 weeks & 10 & $\mathrm{H}$ & Left thalamus & $\begin{array}{l}\text { Upper } \\
\text { extremity }\end{array}$ & Numbness \\
\hline 33 & M & 31 & 2 months & 12 & I & Right frontal lobe & $\begin{array}{l}\text { Hemibody } \\
\text { with face }\end{array}$ & $\begin{array}{r}\text { Lacerating } \\
\text { shooting }\end{array}$ \\
\hline 34 & M & 39 & $\begin{array}{l}2 \text { months and } \\
3 \text { weeks }\end{array}$ & 5 & $\mathrm{H}$ & Left basal ganglia & $\begin{array}{l}\text { Upper } \\
\text { extremity }\end{array}$ & $\begin{array}{r}\text { Numbness, } \\
\text { lacerating }\end{array}$ \\
\hline 35 & $\mathrm{~F}$ & 33 & 2 weeks & 7 & I & Left basal ganglia & $\begin{array}{l}\text { Hemibody } \\
\text { without face }\end{array}$ & $\begin{array}{l}\text { Squeezing, } \\
\text { burning }\end{array}$ \\
\hline
\end{tabular}


Table 1 continued

\begin{tabular}{|c|c|c|c|c|c|c|c|c|}
\hline No. & Gender & $\begin{array}{l}\begin{array}{l}\text { Age } \\
\text { (years) }\end{array}\end{array}$ & $\begin{array}{l}\text { Time since } \\
\text { injury }\end{array}$ & $\begin{array}{l}\text { Pain } \\
\text { duration } \\
\text { (days) }\end{array}$ & $\begin{array}{l}\text { Type of } \\
\text { stroke }\end{array}$ & Lesion & Pain location & $\begin{array}{l}\text { Quality of } \\
\text { pain }\end{array}$ \\
\hline 36 & $\mathrm{M}$ & 58 & $\begin{array}{l}4 \text { months and } \\
1 \text { week }\end{array}$ & 5 & I & $\begin{array}{l}\text { Right basal } \\
\text { ganglia }\end{array}$ & $\begin{array}{l}\text { Hemibody } \\
\text { with face }\end{array}$ & $\begin{array}{l}\text { Burning, } \\
\text { shooting }\end{array}$ \\
\hline 37 & $\mathrm{~F}$ & 43 & $\begin{array}{l}3 \text { months and } \\
2 \text { weeks }\end{array}$ & 9 & I & $\begin{array}{l}\text { Right basal } \\
\text { ganglia }\end{array}$ & $\begin{array}{l}\text { Upper } \\
\text { extremity }\end{array}$ & $\begin{array}{l}\text { Tingling, } \\
\text { numbness }\end{array}$ \\
\hline 38 & $\mathrm{M}$ & 36 & $\begin{array}{c}2 \text { months and } \\
3 \text { weeks }\end{array}$ & 8 & $\mathrm{H}$ & Left basal ganglia & $\begin{array}{l}\text { Upper } \\
\text { extremity }\end{array}$ & $\begin{array}{c}\text { Numbness, } \\
\text { burning }\end{array}$ \\
\hline
\end{tabular}

$M$ male, $F$ female, $H$ hemorrhagic, $I$ ischemic

day $(P<0.001$, Cohen's $d=1.674)$. As time went on, scores increased slightly, but were still significantly lower at the end point $(P<0.001$, Cohen's $d=1.714)$. In contrast, there were no significant differences in the sham group $\left(P=0.067, \eta^{2}=0.129\right)$. The interaction between time and intervention was significant $\left(P<0.001, \eta^{2}=0.669\right)$. The SF-MPQ-2 scores in the rTMS group were significantly lower than in the sham group on the seventh day $(P=0.003$, Cohen's $d=0.771)$, after 2 weeks $(P=0.011$, Cohen's $d=0.650)$, and after 3 weeks $(P=0.027$, Cohen's $d=0.550)$ (Table 2, Fig. 4b).

\section{Secondary Outcome}

All secondary outcomes were measured or recorded before treatment (T0) and on the third week (T4).

\section{Mood Changes: HAM-A and HAM-D}

The HAM-A score in the rTMS group showed a significant reduction after 3 weeks of treatment when compared with baseline $(P=0.010$, Cohen's $d=0.661)$. Similarly, the HAM-D score showed a borderline significant reduction in the rTMS group $(P=0.074$, Cohen's $d=0.435)$. However, neither score showed any significant differences in the sham group or between groups (Table 2, Fig. 5a, b).

\section{Biochemical Changes: Serum BDNF Levels}

Serum BDNF levels were significantly higher after 3 weeks of real magnetic stimulation in the rTMS group $(P<0.001$, Cohen's $d=-0.619)$. In contrast, the difference was only borderline significant in the sham group $(P=0.079$, Cohen's $d=0.428$ ). At the end of the intervention, serum BDNF concentrations in the rTMS group $(115.14 \pm 22.50 \mathrm{pg} / \mathrm{ml})$ were significantly higher than in the sham group $(97.55 \pm 27.63 \mathrm{pg} / \mathrm{ml}) \quad(P=0.048, \quad$ Cohen's $d=-0.487$ ) (Table 2, Fig. 6).

\section{Changes in Neurophysiological Parameters}

RMT The RMT in both the rTMS and sham groups showed significant pre-post effects after 3 weeks of intervention $(P=0.001, P=0.041)$. Intergroup comparison at the end point showed a borderline significant difference between the two groups $(P=0.094$, Cohen's $d=0.936)$ (Table 2, Fig. 7a).

MEP Latency Although both the rTMS and sham groups showed a slight reduction in latency after 3 weeks of treatment, the differences were not significant $(P=0.114, P=0.280$, respectively). Similarly, at the end of treatment, the latency values between the two groups did not show any significant difference $(P=0.972$, Cohen's $d=0.019$ ) (Table 2, Fig. 7b).

Maximal MEP Amplitude The maximal MEP amplitude showed a borderline significant difference after real stimulation $(P=0.064$, 
Table 2 Treatment outcomes between groups

\begin{tabular}{lllll}
\hline Treatment & $\begin{array}{l}\text { Mean }(\mathrm{SD}) \text { before } \\
\text { treatment }\left(\mathrm{T}_{0}\right)\end{array}$ & $\begin{array}{l}\text { Mean }(\mathrm{SD}) \text { after } \\
\text { treatment }\left(\mathrm{T}_{4}\right)\end{array}$ & $\begin{array}{l}\text { Percentage of mean } \\
\text { change }\left(\mathrm{T}_{0} \text { to } \mathrm{T}_{4}\right)\end{array}$ & $\begin{array}{l}\text { Cohen's } d(\text { Sham to } P \text { value } \\
\left.\text { rTMS at } \mathrm{T}_{4}\right)\end{array}$ \\
\hline
\end{tabular}

Primary outcomes

Pain relief after 3 weeks of treatment

Pain scores on NRS

\begin{tabular}{|c|c|c|c|c|c|}
\hline Sham & $5.21(1.03)$ & $5.16(0.96)$ & $-0.49(8.25)$ & & \\
\hline rTMS & $5.26(0.99)$ & $4.10(0.66)$ & $-20.29(16.19)$ & 0.860 & 0.001 \\
\hline \multicolumn{6}{|c|}{ Pain scores on SF-MPQ2-CN } \\
\hline Sham & $42.63(12.33)$ & $43.53(11.92)$ & $3.02(10.26)$ & & \\
\hline rTMS & $43.89(14.48)$ & $33.63(11.59)$ & $-22.25(12.74)$ & 0.550 & 0.027 \\
\hline
\end{tabular}

Secondary outcomes

Mood changes after 3 weeks of treatment

HAM-A

$\begin{array}{llllll}\text { Sham } & 12.89(3.38) & 12.95(3.39) & 0.86(9.06) & & \\ \text { rTMS } & 12.58(3.15) & 11.94(3.08) & -4.93(7.88) & 0.224 & 0.341 \\ \text { HAM-D } & & & & & \\ \text { Sham } & 13.21(2.94) & 12.95(2.80) & -1.52(8.59) & 0.217 & 0.356 \\ \text { rTMS } & 12.95(3.12) & 12.16(3.04) & -5.13(15.18) & \end{array}$

Biochemical modulator changes after 3 weeks of treatment

Serum BDNF concentrations

$\begin{array}{lrrrrr}\text { Sham } & 93.47(26.58) & 97.55(27.63) & 4.65(11.26) & & \\ \text { rTMS } & 93.20(25.26) & 115.14(22.50) & 27.12(19.19) & -0.487 & 0.048\end{array}$

Neurophysiological parameter changes after 3 weeks of treatment $(N=15)$

MEP threshold

\begin{tabular}{|c|c|c|c|c|c|}
\hline Sham & $70.57(7.00)$ & $65.29(7.89)$ & $-7.40(7.67)$ & & \\
\hline rTMS & $69.75(7.53)$ & $56.37(10.73)$ & $-19.51(9.21)$ & 0.936 & 0.094 \\
\hline \multicolumn{6}{|c|}{ MEP latency } \\
\hline Sham & $25.84(4.72)$ & $25.01(4.01)$ & $-2.65(7.10)$ & & \\
\hline rTMS & $26.04(4.12)$ & $25.09(3.82)$ & $-3.34(5.59)$ & 0.019 & 0.972 \\
\hline \multicolumn{6}{|c|}{ MEP maximal amplitude } \\
\hline Sham & $320.98(283.34)$ & $345.74(287.04)$ & $17.62(26.13)$ & & \\
\hline rTMS & $300.52(315.80)$ & $366.31(367.58)$ & $31.26(33.79)$ & -0.062 & 0.907 \\
\hline
\end{tabular}




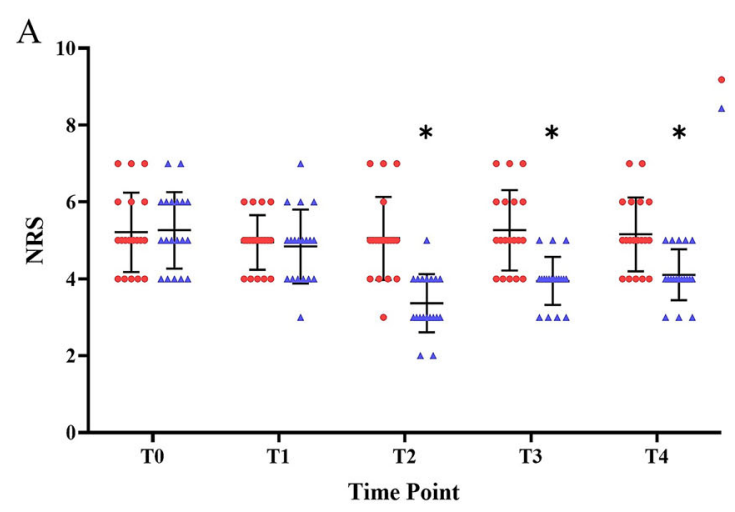

Fig. 4 Pain relief at different time points in the rTMS and sham groups. a Mean NRS score change in the two groups. b SF-MPQ-2 score change in the two groups. Error bars

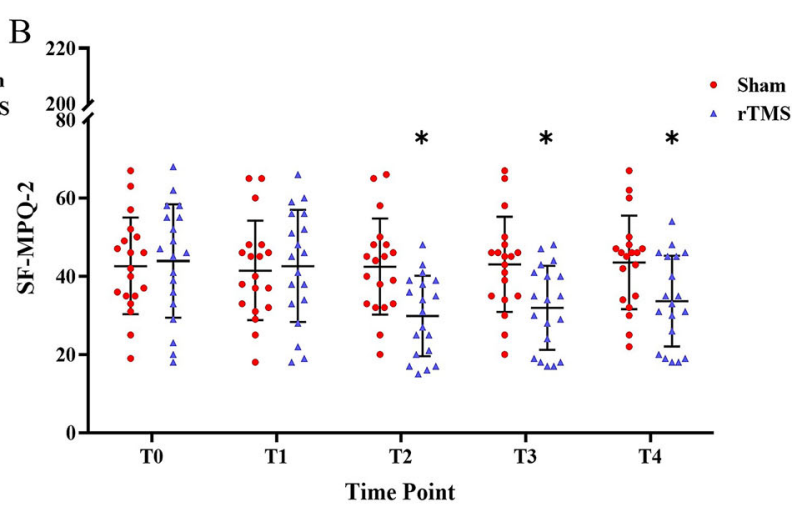

indicate standard deviation (SD). Asterisks indicate significant pre-post differences (with respect to T0) $\left({ }^{*} P<0.05\right)$

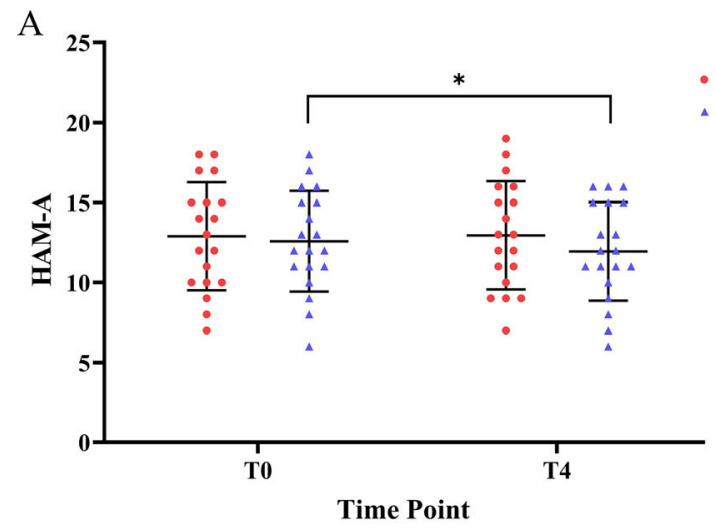

Fig. 5 Mood changes in the rTMS and sham groups at T0 and T4. a Mean HAM-A score changes in the two groups, b HAM-D score changes in the two groups. Error bars indicate standard deviation (SD). Asterisks indicate

Cohen's $d=-0.778)$, but did not change in the sham group $(P=0.154$, Cohen's $d=-0.616)$. Compared with the sham group, the maximal MEP amplitude increased by $77.41 \%(P=0.907$, Cohen's $d=-0.062$ ) in the rTMS group (Table 2, Fig. 7c).

\section{DISCUSSION}

We report here the first study to assess the effects of high-frequency $(10 \mathrm{~Hz})$ rTMS over the primary motor cortex in the affected hemisphere of patients with acute CPSP. In terms of
B

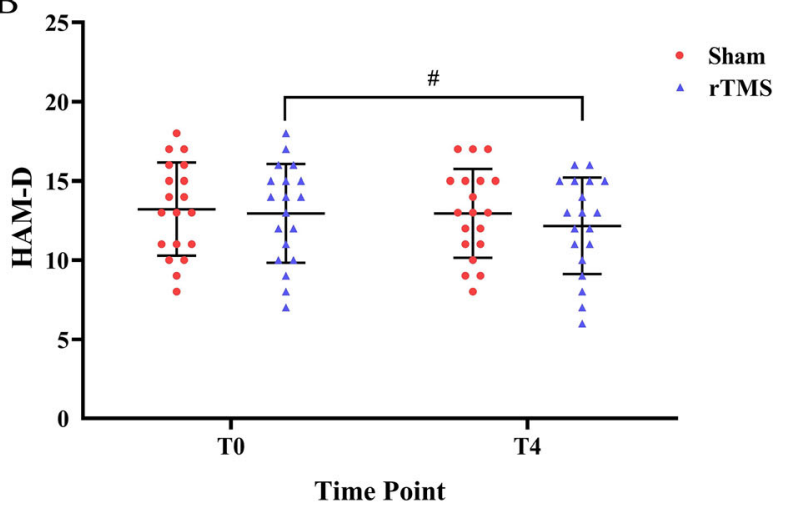

significant pre-post differences (with respect to T0) $\left({ }^{*} P<0.05\right)$. A pound sign (\#) indicates a borderline significant difference compared with T0 $\left(0.05<{ }^{\#} P<0.10\right)$

primary outcomes, the pain intensity decreased from the first to the third week in the rTMS group, but we did not observe this reduction in the sham group. On the other hand, our results demonstrated that rTMS also reduced the HAM$\mathrm{D}$ and HAM-A scores with respect to baseline. Finally, this analgesic effect of rTMS was accompanied by increased levels of BDNF and enhanced cortical excitability parameters in the third week. To our knowledge, this is the first study to evaluate the effectiveness of high-frequency rTMS in patients with acute CPSP. 


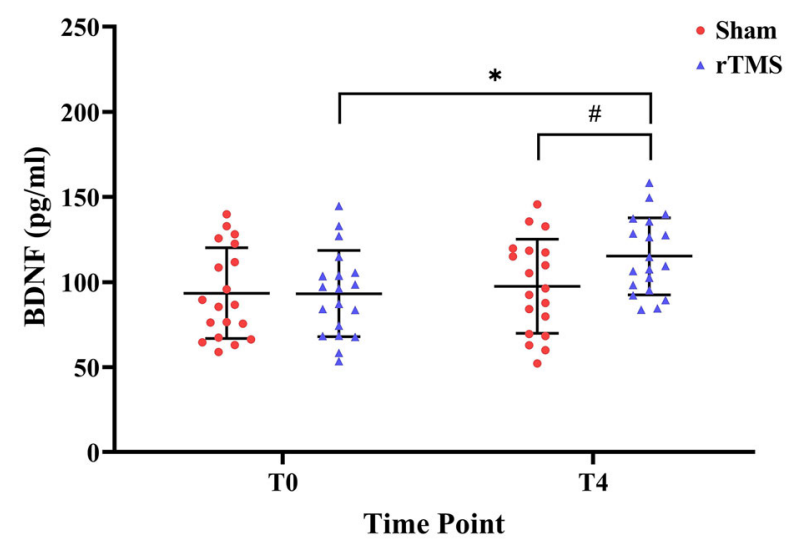

Fig. 6 Mean serum BDNF concentrations at T0 and T4. Error bars indicate standard deviation (SD). Asterisks indicate significant differences with respect to T0 or between the two groups $\left({ }^{*} P<0.05\right)$. A pound sign indicates a borderline significant difference compared with T0 $\left(0.05<{ }^{\#} P<0.10\right)$

The primary outcome of this study was the analgesic effect of rTMS on acute CPSP. We found that patients receiving high-frequency rTMS experienced more pain relief than the sham group. More specifically, high-frequency rTMS $(10 \mathrm{~Hz})$, delivered over a total of 2000 pulses to the upper extremity area of M1 at 80\% RMT, showed a significant pain-alleviating effect in CPSP patients in the first week that lasted until the third week. Until now, the total number of stimuli delivered in most studies on neuropathic pain has exceeded 1000 pulses. Apparently, more stimuli in one treatment session produced better analgesic effects, according to previous studies [27-29]. A few studies opted for less stimulation $(\leq 1000)$ as treatment parameter, but the results were controversial. Hosomi et al. demonstrated that 500 stimulations in a session could effectively relieve CPSP [30], but Kang et al. reported only questionable pain reduction after 3 weeks of administering 1000 stimuli per session for 5 consecutive days (total, 5000 stimuli) [31]. Hence, further investigations are needed to determine the optimal parameters for stimulation.

Our previous randomized controlled trial (RCT), which was intended to investigate the analgesic effects of rTMS specifically on neuropathic pain in patients with SCI [18], revealed that $10 \mathrm{~Hz}$ rTMS produced transient analgesic effects from the third day to the first week. In the present study, using the same parameters as the previous trial, the pain intensity was lowest on the seventh day, and this analgesic effect lasted until the third week. Although the pain scores increased slightly, this changing trend in the analgesic effect was similar to that reported by others. For example, Kobayashi et al. [32] reported that rTMS once a week for 12 weeks induced sustainable long-term relief of CPSP (61.1\% efficacy), although visual analog scale (VAS) scores also rebounded. Future studies are needed to determine whether rTMS has different effects on other types of central nervous system injuries. A slight placebo effect was seen in this study, which was also observed in our previous study. The placebo effect is very common in rTMS studies, and much of the reason is that patients in both groups do not experience exactly the same sensations. Although the coil
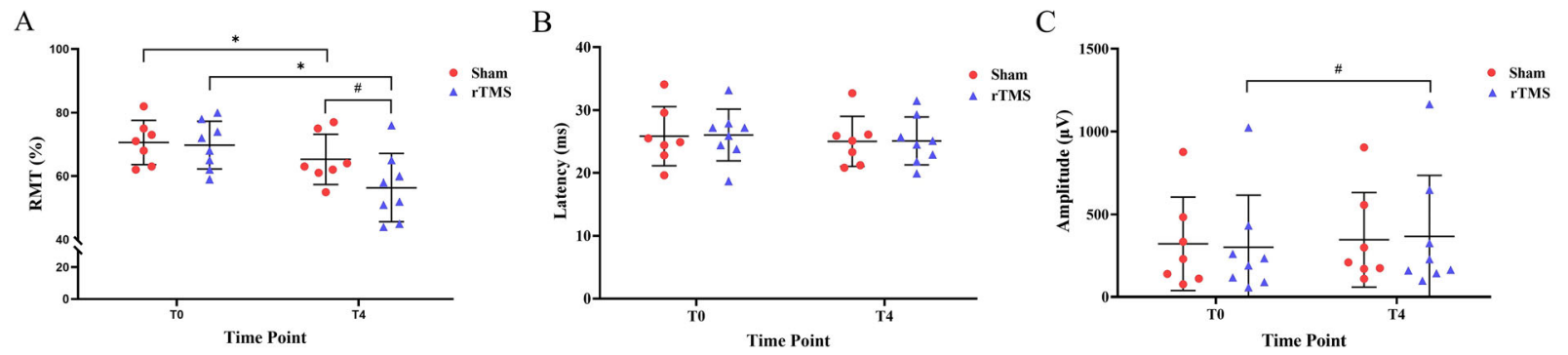

Fig. 7 Changes in neurophysiological parameters. Mean RMT (a), MEP latency (b), and MEP amplitude (c) were recorded at T0 and T4. Error bars indicate standard deviation (SD). Asterisks indicate significant pre-post

differences (with respect to T0) $\left({ }^{*} P<0.05\right)$. A pound sign indicates a borderline significant difference with respect to T0 or between the two groups $\left(0.05<{ }^{\#} P<0.10\right)$ 
used in the sham group had the same shape and mimicked the same sound as the real rTMS coil, patients could find differences as the treatment progressed. In this regard, Diefenbach et al. used a sham coil which mimicked the scalp sensations produced by the real rTMS coil [33]. Although this method is far from perfect, it may be the best available technique to use in the sham group.

In this study, we found that real stimulation of M1 improved depression and anxiety in the rTMS group. However, the HAM-D and HAM-A scores did not differ significantly between the two groups at endpoint. A number of possible underlying reasons may account for this: Firstly, depression and anxiety are partly associated with CPSP; since rTMS effectively alleviated pain, the HAM-D and HAM-A scores correspondingly decreased in the rTMS group. Secondly, assessment of depression and anxiety not only measures effects caused by CPSP, but also measures other emotions which may be regulated by the dorsolateral prefrontal cortex (DLPFC), not by M1. Lastly, most patients enrolled in this study (24 out of 28) did not meet the criteria for diagnosis of post-stroke depression (HAM-D > 17), indicating that depressive symptoms in these patients were relatively mild, so depression and anxiety score improvements would not be that obvious.

BDNF is a "classical" neurotrophic factor which has a close relationship with neuronal plasticity and neuropathic pain. We found that serum BDNF levels increased significantly after 3 weeks of rTMS treatment in patients with CPSP, a finding that is in agreement with our previous work and with other studies. In fact, several studies have indicated that serum BDNF levels correlate closely with the analgesic effects of rTMS. Cheeran et al. reported that high BDNF levels can reduce acute pain in healthy male volunteers, a finding along the same line of our results [34]. In this study, we found a negative correlation between serum BDNF levels and pain scores. However, more trials are needed to clarify whether upregulated serum BDNF levels can decrease pain intensity.

Although the mechanisms underlying the analgesic effects of rTMS on M1 are not well known, previous functional magnetic resonance imaging (fMRI) studies have shown that magnetic stimulation of this brain area can regulate the excitability of subcortical and cortical structures related to pain modulation [35]. Although other cortical structures have been targeted, such as DLPFC and the secondary somatosensory cortex (S2) [36, 37], M1 is the area most commonly associated with analgesic effects [13]. There are three main mechanisms which may explain the analgesic effects of rTMS. First, rTMS may improve the excitability of the cerebral cortex, leading to top-down activation of the brainstem and descending inhibition [38]. Second, rTMS may enhance secretion of neurotransmitters such as BDNF and nerve growth factor (NGF). [27]. Third, rTMS may activate emotional and sensory control areas associated with pain [36]. The RMT is an indicator that reflects the excitability of the cerebral cortex and corticospinal tract (CST) $[24,39]$, as previous pharmacological and physiological studies have demonstrated. In this study, we found a positive correlation between the analgesic effects of rTMS and RMT: as pain intensity decreased, the RMT of the patients at the end of the third week decreased as well, so this could be an important mechanism underlying the analgesic effects of rTMS. On the other hand, MEP is a relatively objective method for evaluating the functionality of the central motor conduction pathways, especially of the corticospinal tract. MEP latency and amplitude serve as indicators, with shorter latencies and larger amplitudes reflecting higher excitability of the motor cortex, and vice versa. Our study found only a borderline significant difference in MEP amplitude in the rTMS group with respect to baseline. However, the MEP latency between the two groups did not differ significantly. These results suggest that MEP latency may be more stable than MEP amplitude, an observation that has been reported previously [40].

The main limitation of our study is the relatively small number of participants. Inadequate sample size may increase the risk of typeII error. Second, we did not classify the participants based on the type of stroke, such as hemorrhagic and ischemic, nor on the affected area. In the future, we would like to evaluate the 
analgesic effects of rTMS on hemorrhagic versus ischemic stroke, and also conduct subgroup analysis between patients with lesions in the thalamus and other brain areas. Third, it is unknown whether the analgesic effects of rTMS on patients with CPSP are long-lasting, due to lack of follow-up.

\section{CONCLUSIONS}

Noninvasive, high-frequency $(10 \mathrm{~Hz})$ rTMS applied over the affected motor cortex may be an effective way of decreasing the intensity of CPSP in the early stages. rTMS may also relieve depression and anxiety. These effects were transient and may be associated with enhanced cortical excitability and increased secretion of BDNF. Thus, rTMS is a safe and effective adjuvant therapeutic tool for managing the early stages of CPSP. Future investigations using functional near-infrared spectroscopy (fNIRS) and functional magnetic resonance imaging (fMRI) may help clarify the precise mechanisms of action of rTMS.

\section{ACKNOWLEDGEMENTS}

We thank Yuan Shen for the helping in statistical analysis and we also thank all the study participants and their families.

Funding. The study and the journal's Rapid Service Fee were supported by grants from the National Natural Science Foundation (81100932, 82072534), the Shaanxi Science and Technology Foundation (2021JM-232, 2020KW050).

Authorship. All named authors meet the International Committee of Medical Journal Editors (ICMJE) criteria for authorship for this article, take responsibility for the integrity of the work as a whole, and have given their approval for this version to be published.

Authors' Contributions. Conception and design: Hua Yuan, Xiang Mou. Acquisition of data: Chen-Guang Zhao, Wei Sun. Statistical analysis and interpretation of data: ChenGuang Zhao, Wei Sun, Fen Ju, Shan Jiang, Hong Wang, Xiao-Long. Drafting the article: ChenGuang Zhao, Wei Sun. Critically revising the article: Hua Yuan, Chen-Guang Zhao, Wei Sun. Reviewed submitted version of manuscript: all authors. Approved the final version of the manuscript on behalf of all authors: Hua Yuan. All authors had full access to all of the data in this study and take complete responsibility for the integrity of the data and accuracy of the data analysis.

Disclosures. Chen-Guang Zhao and Hua Yuan received research grant support from National Natural Science Foundation and Shaanxi Science and Technology Foundation. Hua Yuan is the Director of the Department of Rehabilitation Medicine of Xijing Hospital, Fourth Military Medical University. Wei Sun, Fen Ju, Shan Jiang, Hong Wang, Xiao-Long Sun and Xiang Mou have nothing to disclose.

Compliance with Ethics Guidelines. This work was approved by the Institute Ethics Committee of Xijing Hospital, Fourth Military Medical University (KY20162079). All procedures performed in studies involving human participants were in accordance with the ethical standards of the institutional and/or national research committee and with the 1964 Helsinki Declaration and its later amendments or comparable ethical standards. Informed consent was received to participate. Written informed consent was received from the patients for the publication of the data. We also received written informed consent from the patients for the photograph included in this paper.

Data Availability. The data sets generated during and/or analyzed during the current study are available from the corresponding author on reasonable request.

Open Access. This article is licensed under a Creative Commons Attribution-NonCommercial 4.0 International License, which permits any non-commercial use, sharing, adaptation, distribution and reproduction in any medium or format, as long as you give appropriate credit 
to the original author(s) and the source, provide a link to the Creative Commons licence, and indicate if changes were made. The images or other third party material in this article are included in the article's Creative Commons licence, unless indicated otherwise in a credit line to the material. If material is not included in the article's Creative Commons licence and your intended use is not permitted by statutory regulation or exceeds the permitted use, you will need to obtain permission directly from the copyright holder. To view a copy of this licence, visit http://creativecommons.org/licenses/by$\mathrm{nc} / 4.0 /$.

\section{REFERENCES}

1. Murray CJ, Lopez AD. Mortality by cause for eight regions of the world: Global Burden of Disease Study. Lancet. 1997;349(9061):1269-76.

2. Akyuz G, Kuru P. Systematic review of central post stroke pain: what is happening in the central nervous system? Am J Phys Med Rehabil. 2016;95(8): 618-27.

3. Hansen AP, Marcussen NS, Klit H, Andersen G, Finnerup NB, Jensen TS. Pain following stroke: a prospective study. Eur J Pain. 2012;16(8):1128-36.

4. Osama A, Abo Hagar A, Elkholy S, Negm M, Abd ElRazek R, Orabi M. Central post-stroke pain: predictors and relationship with magnetic resonance imaging and somatosensory evoked potentials. Egypt J Neurol Psychiatr Neurosurg. 2018;54(1):40.

5. Vukojevic Z, Dominovic Kovacevic A, Peric S, Grgic S, Bjelica B, Basta I, et al. Frequency and features of the central poststroke pain. J Neurol Sci. 2018;391: $100-3$.

6. Dube JA, Mercier C. Effect of pain and pain expectation on primary motor cortex excitability. Clin Neurophysiol. 2011;122(11):2318-23.

7. Flaster M, Meresh E, Rao M, Biller J. Central poststroke pain: current diagnosis and treatment. Top Stroke Rehabil. 2013;20(2):116-23.

8. Klit H, Finnerup NB, Andersen G, Jensen TS. Central poststroke pain: a population-based study. Pain. 2011;152(4):818-24.

9. Ramger BC, Bader KA, Davies SP, Stewart DA, Ledbetter LS, Simon CB, et al. Effects of non-invasive brain stimulation on clinical pain intensity and experimental pain sensitivity among individuals with central post-stroke pain: a systematic review. J Pain Res. 2019;12:3319-29.

10. Attal N. Pharmacological treatments of neuropathic pain: the latest recommendations. Rev Neurol (Paris). 2019;175(1-2):46-50.

11. Paul G. Acute neuropathic pain: diagnosis and treatment. Curr Opin Anaesthesiol. 2008;21(5): 590-5.

12. Moisset $\mathrm{X}$, Bouhassira D, Couturier JA, Alchaar H, Conradi S, Delmotte $\mathrm{MH}$, et al. Pharmacological and non-pharmacological treatments for neuropathic pain: systematic review and French recommendations. Rev Neurol (Paris). 2020;176(5): 325-52.

13. Hosomi K, Seymour B, Saitoh Y. Modulating the pain network-neurostimulation for central poststroke pain. Nat Rev Neurol. 2015;11(5):290-9.

14. Lefaucheur JP, Aleman A, Baeken C, Benninger DH, Brunelin J, Di Lazzaro V, et al. Evidence-based guidelines on the therapeutic use of repetitive transcranial magnetic stimulation (rTMS): an update (2014-2018). Clin Neurophysiol. 2020;131(2):474-528.

15. Lefaucheur JP, Andre-Obadia N, Antal A, Ayache SS, Baeken C, Benninger DH, et al. Evidence-based guidelines on the therapeutic use of repetitive transcranial magnetic stimulation (rTMS). Clin Neurophysiol. 2014;125(11):2150-206.

16. Dall'Agnol L, Medeiros LF, Torres IL, Deitos A, Brietzke A, Laste G, et al. Repetitive transcranial magnetic stimulation increases the corticospinal inhibition and the brain-derived neurotrophic factor in chronic myofascial pain syndrome: an explanatory double-blinded, randomized, shamcontrolled trial. J Pain. 2014;15(8):845-55.

17. Mirowska-Guzel D, Gromadzka G, Seniow J, Lesniak M, Bilik M, Waldowski K, et al. Association between BDNF-196 G>A and BDNF-270 C>T polymorphisms, BDNF concentration, and rTMS-supported long-term rehabilitation outcome after ischemic stroke. NeuroRehabilitation. 2013;32(3):573-82.

18. Zhao CG, Sun W, Ju F, Wang H, Sun XL, Mou X, et al. Analgesic effects of directed repetitive transcranial magnetic stimulation in acute neuropathic pain after spinal cord injury. Pain Med. 2020;21(6): 1216-23.

19. Dworkin RH, Turk DC, Farrar JT, Haythornthwaite JA, Jensen MP, Katz NP, et al. Core outcome measures for chronic pain clinical trials: IMMPACT recommendations. Pain. 2005;113(1-2):9-19. 
20. Dworkin RH, Turk DC, Revicki DA, Harding G, Coyne KS, Peirce-Sandner S, et al. Development and initial validation of an expanded and revised version of the Short-form McGill Pain Questionnaire (SF-MPQ-2). Pain. 2009;144(1-2):35-42.

21. Hamilton M. The assessment of anxiety states by rating. Br J Med Psychol. 1959;32:50-5.

22. Hamilton M. A rating scale for depression. J Neurol Neurosurg Psychiatry. 1960;23:56-62.

23. Riedel M, Möller HJ, Obermeier M, SchennachWolff R, Bauer M, Adli M, et al. Response and remission criteria in major depression-a validation of current practice. J Psychiatr Res. 2010;44(15): 1063-8.

24. Du J, Hu J, Hu J, Xu Q, Zhang Q, Liu L, et al. Aberrances of cortex excitability and connectivity underlying motor deficit in acute stroke. Neural Plast. 2018;2018:1318093.

25. Fu J, Zeng M, Shen F, Cui Y, Zhu M, Gu X, et al. Effects of action observation therapy on upper extremity function, daily activities and motion evoked potential in cerebral infarction patients. Medicine. 2017;96(42):

26. Meng Y, Zhang D, Hai H, Zhao YY, Ma YW. Efficacy of coupling intermittent theta-burst stimulation and $1 \mathrm{~Hz}$ repetitive transcranial magnetic stimulation to enhance upper limb motor recovery in subacute stroke patients: a randomized controlled trial. Restor Neurol Neurosci. 2020;38(1):109-18.

27. Attal N, Ayache SS, Ciampi De Andrade D, Mhalla A, Baudic S, Jazat F, et al. Repetitive transcranial magnetic stimulation and transcranial direct-current stimulation in neuropathic pain due to radiculopathy: a randomized sham-controlled comparative study. Pain. 2016;157(6):1224-31.

28. Khedr EM, Kotb HI, Mostafa MG, Mohamad MF, Amr SA, Ahmed MA, et al. Repetitive transcranial magnetic stimulation in neuropathic pain secondary to malignancy: a randomized clinical trial. Eur J Pain. 2015;19(4):519-27.

29. Shu-Min M, Jia-Xiang N, Xuan-Ying L, Li-Qiang Y, Yu-Na G, Yuan-Zhang T. High-frequency repetitive transcranial magnetic stimulation reduces pain in postherpetic neuralgia. Pain Med. 2015;16(11): 2162-70.

30. Hosomi K, Sugiyama K, Nakamura Y, Shimokawa T, Oshino S, Goto Y, et al. A randomized controlled trial of 5 daily sessions and continuous trial of 4 weekly sessions of repetitive transcranial magnetic stimulation for neuropathic pain. Pain. 2020;161(2):351-60.

31. Kang BS, Shin HI, Bang MS. Effect of repetitive transcranial magnetic stimulation over the hand motor cortical area on central pain after spinal cord injury. Arch Phys Med Rehabil. 2009;90(10): 1766-71.

32. Kobayashi M, Fujimaki T, Mihara B, Ohira T. Repetitive transcranial magnetic stimulation once a week induces sustainable long-term relief of central poststroke pain. Neuromodulation. 2015;18(4): 249-54.

33. Diefenbach GJ, Bragdon L, Goethe JW. Treating anxious depression using repetitive transcranial magnetic stimulation. J Affect Disord. 2013;151(1): 365-8.

34. Cheeran B, Talelli P, Mori F, Koch G, Suppa A, Edwards $\mathrm{M}$, et al. A common polymorphism in the brain-derived neurotrophic factor gene (BDNF) modulates human cortical plasticity and the response to rTMS. J Physiol. 2008;586(23):5717-25.

35. Mylius V, Borckardt JJ, Lefaucheur JP. Noninvasive cortical modulation of experimental pain. Pain. 2012;153(7):1350-63.

36. Lindholm P, Lamusuo S, Taiminen T, Pesonen U, Lahti A, Virtanen A, et al. Right secondary somatosensory cortex-a promising novel target for the treatment of drug-resistant neuropathic orofacial pain with repetitive transcranial magnetic stimulation. Pain. 2015;156(7):1276-83.

37. Nardone R, Holler Y, Langthaler PB, Lochner P, Golaszewski S, Schwenker K, et al. rTMS of the prefrontal cortex has analgesic effects on neuropathic pain in subjects with spinal cord injury. Spinal Cord. 2017;55(1):20-5.

38. Garcia-Larrea L, Peyron R. Motor cortex stimulation for neuropathic pain: from phenomenology to mechanisms. NeuroImage. 2007;37:S71-9.

39. Birchenall J, Teremetz M, Roca P, Lamy JC, Oppenheim C, Maier MA, et al. Individual recovery profiles of manual dexterity, and relation to corticospinal lesion load and excitability after stroke-a longitudinal pilot study. Neurophysiol Clin. 2019;49(2):149-64.

40. Elisa K, Minna P, Laura S, Petro J. Onset latency of motor evoked potentials in motor cortical mapping with neuronavigated transcranial magnetic stimulation. Open Neurol J. 2015;9:62-9. 